\title{
Internationalisierung der Translationswissenschaft? Zum Aktualitätsdefizit der De-Westernizing- -Debatte
}

\section{Internationalization of Translation Studies? On Some Shortcomings of the De-Westernizing Debate}

\author{
Lavinia Heller
}

\begin{abstract}
Under the banner of the international turn and De-Westernization, a lively discussion of fundamental questions has been taking place in recent years. The point of departure of this debate is a concern that internal developments in the politics of language and translation have led to a conceptual homogeneity within the specialist discourse of translation studies, with the effect that internationality of the discipline is at stake. The particular provocation of this debate is based primarily on the fact that, ironically, the very discipline known for its linguistic and cultural diversity finds itself being criticized for lacking sensitivity for cultural and linguistic difference at the level of theory and concept-building. The present article discusses the wish to promote disciplinary internationality in the constitution of objects and the formation of concepts, in order to also draw some conclusions about the internationalization of the research community. The aim is to identify a few blind spots within the De-Westernizing debate that hinder the field of translation studies in its fundamental pursuit of internationalizing the discipline.
\end{abstract}

\section{Keywords}

translation studies, De-Westernization, internationalization 
Wissenschaften brauchen für ihre Stabilität zwar einen Bestand unhinterfragter Gewissheiten, für ihre Entwicklung sind sie zugleich aber auch auf die regelmäßige Irritation ihrer selbstverständlichen Annahmen angewiesen. Die Wissenschaftsgeschichte zeigt, dass die damit verbundenen Krisen konstitutiv für den wissenschaftlichen Fortschritt sind, da sie die scientific community in ihrem diskursiven ,Alltagstrott' aufrütteln und zur Revision ihrer begrifflichen und methodologischen Orientierungsmuster, Perspektiven und Problemstellungen motivieren (KUHN 1996; FLECK 1979; POPPER 1972). Häufig werden solche heuristisch produktiven Irritationen durch die Beobachtung disziplinenfremder Diskussionen ausgelöst. So hat die Translationswissenschaft in den letzten Jahren immer neue Zugriffe auf ihren Gegenstand entwickelt, die etwa unter dem programmatischen Schlagwort des cultural turn, social turn, post colonial turn, ideological turn, activist turn etc. firmieren und zur weiteren Ausdifferenzierung der Disziplin und ihrer Forschungsfelder geführt haben. Innovationsimpulse müssen allerdings nicht immer von außen kommen, zumeist entstehen sie durch innerdisziplinäre Diskussionen. So hat der sogenannte De-Westernizing-Diskurs innerhalb der Translationswissenschaft eine lebhafte Grundsatzdiskussion angestoßen, die bereits unter dem Banner des international turns (CHEUNG 2005) geführt wird. ${ }^{1}$ Ausgangspunkt dieser Debatte ist die Sorge, dass sich über die zunehmend und bald ausschließlich englischsprachige Fachdiskussion eine diskursive Homogenität eingestellt hat, vor deren Hintergrund kaum noch von einer Internationalität des Fachs gesprochen werden kann. Die besondere Provokation dieser Debatte gründet in erster Linie darin, dass sich ausgerechnet die für Sprachen- und Kulturenvielfalt ausgewiesene Disziplin mit dem Vorwurf der Unsensibilität für kulturelle und sprachliche Differenzen auf der Ebene der Theorie- und Begriffsbildung konfrontiert sieht. Wir haben es hier also eigentlich nicht mit einem neuen sogenannten turn zu tun, der sich oft lediglich auf Teilgebiete der Disziplin auswirkt, sondern mit einer buchstäblichen Identitätskrise des Fachs als Ganzem. Entsprechend aufgeregt und unsystematisch wird diese Debatte auch geführt. Dabei laufen die Forderungen einer De-Westernization wegen ihres zunehmend normativen und moralischen Duktus Gefahr, zu einem kaum noch kritikfähigen Mantra zu degenerieren und so ihr analytisches Reformpotential einzubüßen. ${ }^{2}$ Die ideologiekritischen Implikationen des Begriffs De-Westernization lassen den Aufstieg zu einer rhetorischen Hochwertformel schon erahnen. Denn De-Westernization verweist mittlerweile nicht nur auf ,Internationalisierung“, sondern auch auf ,Entideologiesierung', ,Antiessentialismus', ,Antipositivismus', ,Anti-Eurozentrismus', ,Hybridisierung und ,Indigenisierung،.

1 Initiiert wurde diese Diskussion 2002 von SUSAM-SARAJEVA mit ihrem Aufsatz A, Multilingual ${ }^{\star}$ and ,International Translation Studies? Prominente Stimmen in diesem Diskurs sind etwa Martha Cheung, Rita Kothari, Sebnem Susam-Sarajeva, Maria Tymoczko und Judy Wakabayashi. Wichtige Beiträge zu dieser Diskussion finden sich außerdem in Heften 7:1 und 7:3 der Zeitschrift Translation Studies und im Sonderheft Eurocentrism in Translation Studies der Fachzeitschrift Translation and Interpreting Studies von 2011.

2 In der Tat hört man kaum kritische Stimmen zu diesem Diskurs. Ausnahmen bilden etwa und Michael BOYDEN 2011, Andrew CHESTERMAN und sein umstrittener Artikel Universalism in Translation Studies, mit dem er die Forumsdiskussion 2014 in den Heften 7:1 und 7:3 der Zeitschrift Translation Studies eröffnet hat. Siehe auch HELLER 2017a. 
Im Folgenden soll versucht werden, eines der Grundanliegen der De-Westernizing-Debatte etwas systematischer zu betrachten, nämlich die Internationalisierung des Fachs. Dieses Anliegen bildet gewissermaßen den Auftakt der Debatte und scheint mir besonders fundamental zu sein, insofern es aus dem Vorwurf der mangelnden Sensibilität der Translationswissenschaft für kulturelle Differenzen entwickelt wurde und damit das Fach als Ganzes in seinem lang tradierten Selbstverständnis getroffen hat. In diesem Sinne hatte ich weiter oben von einer Identitätskrise gesprochen. Das Thema wird auf der Ebene der Gegenstandskonstitution und der Begriffsbildung diskutiert, wobei diese Ebenen allein der Übersichtlichkeit halber getrennt voneinander besprochen werden, denn es wird sich immer wieder zeigen, dass sie aufs engste aufeinander verwiesen bleiben. Im abschließenden Teil werden einige Schlussfolgerungen zur Internationalisierung der Forschungsgemeinschaft gezogen. Ziel ist es, die blinden Flecken der De-Westernizing-Debatte zu identifizieren, die die Translationswissenschaft bei der Verfolgung ihres Grundanliegens der Internationalisierung behindern. Dabei wird auch der Frage nachgegangen, wie eine Internationalisierung auf diesen Ebenen praktisch aussehen könnte. Genau dieses Problem wird nämlich in der Diskussion häufig übergangen oder unter dem unspezifischen Vorschlag abgehandelt, sich für mehr Austausch zwischen den verschiedenen Translationskulturen ${ }^{3}$ zu engagieren.

\section{De-Westernization der Translationswissenschaft}

Einen Hauptgrund für die kritisierte Homogenisierungstendenz innerhalb der Fachdiskussion sehen die De-Westernizer in einer spezifischen Sprach- und Translationspolitik. In der Tat wird, wie auf Fachtagungen anderer Disziplinen auch, auf translationswissenschaftlichen Konferenzen immer seltener in einer anderen Sprache als in den sogenannten ,großen' Wissenschaftssprachen, d.h. auf Englisch, Französisch oder Deutsch vorgetragen, wobei das Englische deutlich überwiegt. ${ }^{4}$ Diese Sprachpolitik wird vor allem auch von Fachjournalen forciert. Die Dominanz der ,großen` europäischen Wissenschaftssprachen wird zudem auch translationspolitisch gefördert. So werden aus vielen ,kleinen‘ Sprachen kaum oder überhaupt keine translationsbezogenen Texte übersetzt,

3 In diesem Artikel wird nicht konsequent zwischen ,Translation“ und ,Übersetzung', ,Translator‘ und ,Übersetzer` bzw. zwischen Translationskultur und Übersetzungskultur (PRUNČ 2008) unterschieden, wie es an anderer Stelle notwendig wäre. Wenn spezifisch das Übersetzen (schriftliche Translation) gemeint ist, erschließt sich dies aus dem Kontext. Es wird außerdem nicht durchgängig zwischen geschlechtsspezifischen Personenbezeichnungen differenziert, wo dies nicht erforderlich ist. Das Maskulinum und das Femininum werden abwechselnd generisch verwendet.

4 Die Unterscheidung von großen und kleinen Wissenschaftssprachen ist allerdings problematisch. Die Geschichte zeigt nämlich, dass zu unterschiedlichen Zeiten andere Sprachen den akademischen Diskurs dominiert bzw. als lingua franca gedient haben. Darüber hinaus dominieren in einem zeitgenössischen, mithin ausdifferenzierten Wissenschaftssystem in unterschiedlichen Fachdiskursen auch unterschiedliche Sprachen. Die Tendenz einer fächerübergreifenden Dominanz des Englischen lässt sich heute freilich nicht leugnen. In den letzten Jahren ist in der Translationswissenschaft mitunter auch die Frage aufgeworfen worden, ob oder welche Möglichkeiten Translatorinnen haben, den mit der Monolingualität der Wissenschaft einhergehenden Homogenisierungstendenzen entgegen zu wirken, siehe dazu vor allem BENNETT (2007 und 2015). 
aus dem ,Zentrum“ hingegen viel translationswissenschaftliches Material in die ,Peripherie' importiert. Dies gilt nicht erst für die aus europäischer Sicht ,exotischen` Translationskulturen, wie den asiatischen, sondern auch für die osteuropäischen Übersetzungskulturen. $^{5}$

Diese sprach- und translationspolitischen Entwicklungen haben zu der Sorge geführt, dass über unidirektionale Übersetzungsprozesse aus den ,großen“ in die ,kleinen“ Wissenschaftssprachen nicht nur eine bestimmte Terminologie, sondern auch ein von ,westlichen“ Übersetzungskulturen tradierter Reflexionsstil übertragen würde, und zwar so, dass sich mit der Zeit eine nicht nur fachsprachliche, sondern auch konzeptionelle Homogenität nach ,westlichem‘ Modell etabliere. Dieser Translationspolitik kann in der Tat ein starker Disseminationseffekt zugesprochen werden, da die ,westlichen' Konzepte nicht erst von Wissenschaftlern zu Forschungszwecken rezipiert werden, sondern über Lehrmaterial auch in der Lehre Verbreitung finden. Als ,westlich“ werden in dieser Debatte diejenigen Übersetzungskulturen charakterisiert, die in der abendländischen Geistesgeschichte verwurzelt sind. ${ }^{6} \mathrm{Zu}$ den für die abendländischen Denkgewohnheiten ,typischen“ translationsbezogenen Vorannahmen zählen in der De-Westernizing-Debatte etwa der Monolingualismus, die Schriftlichkeit und eine repräsentationalistische bzw. Transfer-zentrierte Translationsvorstellung, in deren Mittelpunkt das Original und die (Un-)Möglichkeit seiner Repräsentation bzw. Reproduzierbarkeit stehen. In der Kritik stehen damit die Begriffe: Original(treue), Invarianz, Äquivalenz, Transfer, Repräsentation bzw. Reproduktion. Die Bedenken der De-Westernizer beziehen sich darauf, dass die Verbreitung dieses für typisch westlich gehaltenen Übersetzungsverständnisses der empirischen Vielfalt der Übersetzungsformen nicht gerecht würde. Die Überzeugung dabei ist, dass Translation in unterschiedlichen Sprach- und Kulturräumen andere, z.T. unvergleichbare Realisierungsformen findet. Über eine „normative extension of Eurocentric ideas about translation" (TYMOCZKO 2009) würde jedoch der Forschungsgegenstand der Translationswissenschaft auf eine bestimmte, im Westen tradierte, unter den Bedingungen der Monolingualität, der Schriftlichkeit und einer essentialistischen Sprachauffassung sich entwickelten Übersetzungsform reduziert.

5 Dabei hatte HOLMES bereits 1988, d.h. in der Konsolidierungsphase der Disziplin, im Wissen um die in diesen Sprachen entstandenen theoretisch gehaltvollen Texte in seinem Aufsatz The Future of Translation Theory: A Handful of Theses auf deren Übersetzung gedrängt.

6 Diese plakative Unterscheidung von ,Westen` und ,Nichtwesten' ist fachintern wegen der pauschalisierenden Zusammenfassung eigentlich sehr heterogener Translationskulturen als ,Westen' und ,Nicht-Westen' in Frage gestellt worden. In den letzten Jahren wurde demgegenüber immer häufiger das Begriffspaar ,Zentrum und ,Peripherie" verwendet (vgl. SUSAM-SARAJEVA 2002), da es hier weniger um geographische Positionen als um fachkommunikative Verdichtungsräume und um die sich daraus entwickelnden Geltungsansprüche geht. Doch auch diese Unterscheidung ist problematisch, da das Verhältnis von ,Zentrum “ und "Peripherie“ ständigem Wandel ausgesetzt ist. In diesem Aufsatz wird daher ,Westen“, ,Nicht-Westen“, „Peripherie‘ und ,Zentrum‘ in einfachen Anführungszeichen verwendet, um diese Vorbehalte zu markieren. 


\section{Internationalisierung des Forschungsgegenstandes}

Dass das translationswissenschaftliche ,Zentrum‘ erst so spät ein Bewusstsein über die Historizität und die lokale Gebundenheit von Übersetzungspraktiken gewonnen hat, ist angesichts der Zentralität der Frage der sprachlichen und kulturellen Inkommensurabilität erstaunlich. Diese Verspätung macht wohl auch die identitätskritische Dramatik dieser ,Entdeckung“ aus. Erst in den letzten Jahren hat sich also im ,Zentrum“ ein Interesse für die ,peripheren' Praktiken, etwa an der chinesischen (CHEUNG 2006, CHEUNG/NEATHER 2017; LIU 1995), japanischen (SATO-ROSSBERG/WAKAYABASHI 2012), indischen (WAKAYABASHI/KOTHARI 2009), afrikanischen (INGGS/ MEINTJES 2009; MARAIS 2011) und jüngst auch für die osteuropäischen (SCHIPPEL/ ZWISCHENBERGER 2016) Translationskultur entwickelt mit dem Ansinnen, über einen solchen Einblick die Forschungsagenda zu erweitern. Diese Studien haben zu der Einsicht beigetragen, dass sich unter unterschiedlichen sozialen und kulturellen Rahmenbedingungen jeweils andere translatorische ,Realitäten“ herausgebildet haben.

Entsprechend der Tradition des Fachs, theoretische und angewandte Translationsforschung zu verschränken, sind die Ziele und Zwecke der Orientierung der De-Westernizer an der translatorischen ,Peripherie“ sowohl translationstheoretischer als auch translationspraktischer Natur. Denn es soll nicht nur Einblick in andere Translationskulturen gewonnen werden, um neue Formen der Translation in den Beobachtungsfokus zu bringen und so etwa die Belastbarkeit der im ,Westen' entwickelten Begriffe und Methoden zu prüfen und zu relativieren. ${ }^{7}$ Die Hoffnung zielt darüber hinaus auch darauf ab, Inspiration für die Lösungen aktueller praktischer Probleme zu finden, denen im ,Westen“ keine erkennbare Tradition entspricht (TYMOCZKO 2009). Diese betreffen z.B. das Verhältnis zwischen Ausgangstext (AT) und Zieltext (ZT), das unter den heutigen (Arbeits-)Bedingungen Realisierungsformen erfordert, die dem herkömmlichen ,westlichen' Verständnis von Übersetzung entgegenstehen. Die von modernen Kommunikations- und Translationstechnologien (Internet, E-Mail, CAT-Tools, digitale Textcorpora etc.) unterstützten Arbeitsmodi haben in der Tat zu neuen Formen der Arbeitsteilung geführt, die einen ZT hervorbringen, der weder in einem Invarianzverhältnis zu einem AT definiert, noch einem Akteur allein zugeschrieben werden kann, wie dies im ,westlichen' Translationsdiskurs tradiert ist. Der unter den modernen Arbeitsbedingungen entstandene ZT kann heute häufig nur noch als ,Patchwork، vieler Akteure oder Aktanten (Latour) begriffen werden, das auf entsprechend viele Quellen verweist (AT, Datenbanken, Paralleltextkorpora, Glossare, Rohübersetzungen, Versionen etc.). In dieser Hinsicht besonders radikale Translationsformen finden wir etwa im Bereich der crowd und fan translation, wo eine translatorische Autorschaft kaum mehr identifiziert werden kann (O'HAGAN 2011). ${ }^{8}$ Derartige Herausforderungen rufen nicht zuletzt auch unter den Praktikern allgemeine

7 Dieses rein instrumentelle Interesse am empirischen Material der Peripherie ist bereits Gegenstand der Kritik geworden. Siehe dazu SUSAM-SARAJEVA (2002).

8 Zum kollaborativen Charakter der Translation siehe außerdem ALFER/ZWISCHENBERGER (erscheint 2020); CORDINGLEY/FRIGAU MANNIG (2017), HOLZ-MÄNTTÄRI (1984); JANSEN/WEGENER (2013); RISKU (2014). 
Verunsicherung hervor, da sie nicht dem herkömmlichen ,westlichen', auf ein eindeutiges AT-ZT-Invarianzverhältnis aufbauendes Translationsverständnis entsprechen, das bisher der Orientierung gedient hätte. Maria Tymoczko, eine der führenden Stimmen des Internationalisierungsdiskurses, merkt dazu an:

Not surprisingly, many translators and translation scholars [...] perceive these shifts as threats and sources of anxiety because they seem to undermine all that people have learned about translation as transfer, as well as about the importance of fidelity, the concept of equivalence, the sacredness of the word and so on. (TYMOCZKO 2009: 404)

Die Hoffnung ist also, in den Translationspraktiken anderer Kulturen Inspiration für kreative Lösungen aktueller Probleme zu finden. Dabei wird gerne auf historische Übersetzungskulturen rekurriert, etwa auf die Tradition der translatorischen Teamarbeit bei der Übersetzung buddhistischer Sutren im alten China, ${ }^{9}$ oder auf das Kreativitätspotential der afrikanischen, indischen und arabischen Tradition, die mitunter in einer oralen Übersetzungs- und Erzählkultur gründen und der Translatorin zu früheren Zeiten mehr Authorität und kreative ,Freiheiten` eingeräumt haben soll (ibid.: 410; 2014). In diesem historischen Rückgang manifestiert sich die Befürchtung, dass sich mittlerweile Translationsnormen über die Sprach- und Kulturgrenzen hinweg so weit an die ,westlichen' Standards angepasst haben, dass sich die heutigen Translationspraktiken in vielen sozialen Kontexten im interkulturellen Vergleich zu geringfügig unterscheiden, um sich grundsätzlich neu orientieren zu können (TYMOCZKO 2014: 173ff.). Es ist allerdings zu vermuten, dass dies nicht auf einen forcierten Export ,westlicher' Normen zurückzuführen ist, sondern auf den immer engeren translatorischen Austausch ,westlicher und ,nicht westlicher' Kommunikationsgemeinschaften. Gerade die kulturelle Praxis des Übersetzens kann keinen ,reinen' kulturellen Ursprung haben, da sie immer schon im interkulturellen Kontakt bei der Lösung rekurrierender situationsspezifischer Übersetzungsprobleme entsteht. In diesem Sinne profilieren sich Translationskulturen gerade in der Auseinandersetzung mit ihren Translationspartnern. Der forcierte Historismus des De-Westernizing-Diskurses droht moderne Translationskulturen nicht nur zu exotisieren, sondern sie auch auf ihre überholten Traditionen zurückzudrängen, die in vergangenen interkulturellen Austauschprozessen verwurzelt sind. Im Bemühen die „erosion of translation communities" (TYMOCZKO 2014) aufzuhalten und der westlichen konzeptuellen Dominanz entgegenzuwirken, hat sich die Gewohnheit eingeschliffen, ,natürliche‘ von ,unnatürlichen` Translationspraktiken zu unterscheiden und bei der Zusammenstellung der Daten die heutigen, modernen Translationspraktiken der ,Peripherie‘ als ,uneigentlich`zu übergehen.

Ich möchte hier allerdings auf ein anderes, für die Erfüllung der Forderung, den Forschungsgegenstand zu internationalisieren, grundsätzlicheres methodologisches Problem hinaus. Es besteht in der Annahme, dieser Forderung dadurch nachkommen zu können,

9 Hierbei wird übergangen, dass im europäischen Mittelalter das kollaborative Übersetzen durchaus auch ein verbreitetes Übersetzungsverfahren war, das erst im Zuge der Renaissance und einem sich neu entwickelnden Translationsverständnis zurückgedrängt worden ist (siehe dazu BISTUÉ 2017). 
möglichst viele unterschiedliche translatorische Materialien bzw. Darstellungen verschiedener Translationspraktiken aus aller Welt zusammenzutragen. Forschungsgegenstände werden schließlich nicht einfach vorgefunden, wissenschaftliche Objekte sind vielmehr das Ergebnis objektivierender und selegierender Transformationen, Konstruktionen und Abstraktionen der lebensweltlichen Erfahrung. In diesem Sinne erklärt der Philosoph Hans Blumenberg in seinen begriffshistorischen Überlegungen:

Bevor die Wiese sich in physikalische, chemische und botanische Darstellungskomplexe auflöst, muß ihre lebensweltliche Totalität in einen gewandelten Aspekt überführt und die Art und Weise, sie zu sehen, theoretisch modifiziert worden sein. (BLUMENBERG 2007: 98)

Wenn vom Forschungsgegenstand der Translationswissenschaft gesprochen wird, kann es also nicht darum gehen, Dinge und Sachverhalte aufzuzählen, denn der Gegenstand eines Fachs ist nicht einfach die Summe bestimmter empirischer Phänomene. Etwas wird zum Forschungsgegenstand der Translationswissenschaft, indem es von dieser mit ihren Begriffen und Methoden problematisiert und in einen translationswissenschaftlichen Problemhorizont eingefügt wird. Für die konstruktivistisch geschulte Leserin mag dies zunächst trivial anmuten. Die Selbstverständlichkeit dieser These lässt allerdings oft vergessen, welche Tragweite sie eigentlich für eine tatsächlich internationale Integration des Forschungsgegenstandes hat. Vor diesem Hintergrund hängt die Integration eines Forschungsbereichs nämlich maßgeblich von der wissenschaftlichen Begrifflichkeit ab und nicht schon von der Anzahl möglichst heterogener Beobachtungsmaterialen.

\section{Internationalisierung der Begriffsbildung}

In den anthropologisch motivierten begriffshistorischen Überlegungen seiner Theorie der Unbegrifflichkeit hatte Blumenberg die Analogie zwischen dem Bau einer Falle und der Bildung eines Begriffs bemüht, die beide „aus der actio per distans, aus dem Handeln auf räumliche und zeitliche Entfernung hin entstanden“ sind (BLUMENBERG 2007: 11). Denn in der Konstruktion der Falle muss die Verhaltensweise, die Größe, Figur und das Maß der zu fangenden Beute vorweggenommen werden (ibid.: 10). Ebenso ist der Begriff hinsichtlich des noch zu Erwartenden gebildet, er muss also ein bestimmtes Maß an Unbestimmtheit haben, um noch Unbekanntes erfassen zu können. Ist der Begriff erst die Voraussetzung für wissenschaftliche Wahrnehmungen und Handlungen, dann erweist sich seine Leistung darin, „die Verfügbarkeit des Gegenstandes potentiell bereitzuhalten, abrufbar zu machen“ (BLUMENBERG 2007: 28).

Die berechtigten Bedenken des De-Westernizer sind, dass der derzeitig dominante Translationsbegriff den Gegenstand Translation in seinen mannigfaltigen Erscheinungsformen eben nicht bereithalten und abrufbar machen kann, sondern im Gegenteil durch seine Spezifizität nur einen Ausschnitt davon als translationswissenschaftlich relevant zu selegieren in der Lage ist. Wie weiter oben erklärt wurde, wird dieser Missstand maßgeblich auf eine asymmetrische Translationspolitik innerhalb des Fachdiskurses zurückgeführt, 
der die Verbreitung eines ,westlich“ geprägten Vokabulars fördert. Vergleicht man den für die Translationswissenschaft zentralen Terminus ,Translation“ in unterschiedlichen Fachsprachen, springt tatsächlich gleich eine gewisse metaphorologische Vergleichbarkeit über die Sprachfamiliengrenzen hinweg ins Auge:

\begin{tabular}{|l|l|}
\hline LAT & Translatio \\
\hline IT & Traduzione \\
\hline FR & Traduction \\
\hline RO & Traducere \\
\hline ES & Traducción \\
\hline PT & Tradução \\
\hline EN & Translation \\
\hline DE & Translation \\
\hline RU & TepeBog \\
\hline BKS & Prevođenje \\
\hline PL & Przekład \\
\hline CZ & Preklad \\
\hline
\end{tabular}

Die Vergleichbarkeit dessen, was jeweils als „Translation“ bezeichnet wird, liegt hier in ihrer Konzeption als Transfer (vom lateinischen translatio bzw. transferre) im Sinne von ,Überqueren“, ,Über-setzen', ,Überführen', ,Übertragen“ von Etwas von einem Ort an einen anderen. Dieses Motiv ist jedoch nicht bloß lexikalisch (trans) begründet. Es hängt auch geistesgeschichtlich mit dem historiographischen Konzept der translatio (translatio artium, translatio sapientiae, translatio studii, translatio imperii) zusammen. ${ }^{11}$ Ausgehend vom translatio-Gedanken impliziert der Fachterminus ,Translation“ nicht nur das ,Überqueren“ und ,(Hin-)Übertragen` im konkreten, sondern auch die ,Übertragung، und,Weitergabe‘ im abstrakten Sinne, etwa von Kultur (translatio artium), Bildung und Wissen (translatio sapientiae, translatio studii), von Macht und Heil (translatio imperii). Je tiefer man in die Geschichte eindringt, desto eindeutiger kristallisiert sich die Konstante heraus, die den translatio-Begriff unverändert durch die Jahrhunderte hinweg zu begleiten scheint: die Idee des Transfers und der implizierten Vorstellung der Invarianz dessen, was transferiert wird. Während translatio noch im Mittelalter sowohl die interlinguale Übersetzung als auch die ,Deplatzierung;, d.h. die örtliche Verschiebung etwa von Macht oder Bildung bezeichnet, wird mit den kulturellen und sprachpolitischen Entwicklungen der Renaissance für die romanischen Sprachen eine Unterscheidung getroffen zwischen translatio

10 Entgegen der Annahme, ,Translation' sei als eine Entlehnung des englischen ,translation“ in die deutsche translationswissenschaftliche Terminologie eingeführt worden, verweist VERMEER (2000:537) auf die Substantive ,Translatze und ,transla(t)zion‘, die im deutschen Sprachraum bereits im 15. Jahrhundert auftreten. Der Terminus ,Translation“ wurde 1963 von Kade eigens für die Fachkommunikation als Oberbegriff für das Übersetzen und Dolmetschen (wieder-)eingeführt. Allerdings setzte sich der Terminus erst in den 1980ern und 1990ern wirklich durch.

11 Für eine detaillierte Entwicklung des translatio-Gedankens siehe GOEZ (1958), der vor allem der Frage nach seinem Ursprung und seiner Funktion für die Geschichtsschreibung in unterschiedlichen Diskursen (etwa in der römischen Literatur, in der Bibel, in der kurialen Translationstheorie, in der Geschichtsschreibung der Humanisten und der der Reformation) nachgeht. 
und traductio (traduzione, traduction, traducción, tradução), wobei translatio sich weiter auf die ,Deplatzierung، konkreter Gegenstände und Symbole bezieht und traductio fortan auf das Übersetzen von einer Sprache in die andere verweist. ${ }^{12}$ Die Transfer-Hypothese des translatio-Gedankens hat sich jedoch metaphorologisch in vielen Sprachen in den traductio-Begriff eingeschrieben, wie sich allein schon an den oben aufgeführten Termini ablesen lässt. Es lassen sich jedoch auch Übersetzungskulturen identifizieren, die von einer Konzeption des Übersetzens als Transformation ausgehen. Diese manifestiert sich z.B. in der Metapher des Wendens und Drehens (etwa im Lateinischen ((con)versio, vertere; interpretatio), Altfranzösischen (turner), Spanischen (volver algo), Türkischen (Çeviri), Chinesisch (翻译), Japanischen (翻訳)) oder in der Bedeutung des ,nochmals, aber anders Sagens“ (etwa im Arabischen (ترجمة)) und des ,Transformierens durch eine neue Versprachlichung، (etwa im Niederländischen (vertaling) und zu früheren Zeiten auch im Deutschen (verdeutschen)). Dabei fällt auf, dass sich im Translationsdiskurs einiger Sprach- und Kulturräume erst später eine Transferterminologie etabliert zu haben scheint, wie etwa im Lateinischen, Französischen, Deutschen und Englischen. Aus einer historischen Distanz lässt sich somit die fortschreitende translatorische Verbreitung des Transfervokabulars regelrecht beobachten. Einen besonders deutlichen Ausdruck findet die Übersetzung des Transfer-Vokabulars schließlich in den fachterminologischen Neologismen jüngeren Datums, wie etwa ,Ausgangstext“ und ,Zieltext‘ bzw. ,Ausgangskultur und ,Zielkultur` und ihren ,Äquivalenten` in den sprachlich unterschiedlichsten Fachdiskursen:

\begin{tabular}{|c|c|c|}
\hline IT & testo fonte / partenza & testo di arrivo \\
\hline FR & texte de base / initial/ source / de départ & texte cible / d'arrivée \\
\hline $\mathrm{RO}$ & text sursă & text-țintă \\
\hline ES & texto inicial / fuente / de origen & texto meta / de destino \\
\hline PT & texto inicial / fonte / de origem & texto de destino / chegada \\
\hline EN & source text & target text \\
\hline $\mathrm{DE}$ & Ausgangstext & Zieltext \\
\hline BKS & izvorni tekst & ciljni tekst \\
\hline $\mathrm{PL}$ & tekst źródłowy & tekst docelowy \\
\hline $\mathrm{HU}$ & forrásnyelvi szöveg & célnyelvi szöveg \\
\hline $\mathrm{CN}$ & 原/源文本 (yuan / yuan wenben) & 目的语文本 (mudiyu wenben) \\
\hline JP & \begin{tabular}{|l|} 
原文; 起点テクスト \\
(genbun; kiten tekusuto) \\
\end{tabular} & $\begin{array}{l}\text { 訳文; 目標テクスト } \\
\text { (yakubun; mokuhyō tekusuto) }\end{array}$ \\
\hline AR & النص المصدر (an-Naș al-Mașdaru) & النص الـهدف (an-Naș al-Hadafu) \\
\hline TR & kaynak metin & erek metin \\
\hline
\end{tabular}

12 Der Ausdruck traductio ist in der Bedeutung der interlingualen Übersetzung erstmals beim Humanisten Leonardo Bruni um 1400 belegt (GIPPER 2017: 31). Zur Bedeutung Brunis für die Reflexionsgeschichte der Translation siehe außerdem FOLENA (1991) und MARASSI (2009). Interessant ist in diesem Zusammenhang die Wiederbelebung des Begriffs der traslazione (sic!), die italienische Form von translatio, im italienischen philosophischen Übersetzungsdiskurs. Ein solcher Rückgang zu einem maximal entgrenzten Translationsbegriff ist auch im deutschen kulturwissenschaftlichen Übersetzungsdiskurs zu beobachten. Dort wo Übersetzung auf unterschiedlichste Formen von Transfer- und Transformationsprozessen, Interaktionsmodi und Forschungsmaximen verweist (vgl. BACHMANN-MEDICK 2008; 2014; WAGNER/LUTTER/LETHEN 2012). Siehe DIZDAR (2009) und HELLER (2017b) für eine Kritik an der ,Überdehnung` des Translationsbegriffs. 
Die Einheitlichkeit der Terminuspaare Ausgangstext-Zieltext über die Sprachgrenzen hinweg ist augenfällig. Über die Metaphorik Ausgangs-/Quellen-/Ursprungs(text)-und-Ziel (text) hat sich ein bipolares, Transfer-zentriertes und in diesem Sinne essentialistisches Verständnis von Translation verbreitet. Dieses Vokabular stützt nämlich die Idee, dass im Translationsprozess Bedeutungsinhalte von einem Translator aus dem Ausgangs-, d.h. dem Ursprungstext möglichst vollständig in einen Zieltext über(ge)setzt bzw. transferiert werden könnten. Bemerkenswert ist hier, dass sich diese dichotome Terminologie (AT-ZT) auch in denjenigen Fachsprachen etabliert hat, in denen ,Translation“ eine Transformations- und keine Transfer-Hypothese impliziert. Die fachterminologische Zweipoligkeit der Übersetzungskonzeption überschreibt gewissermaßen die noch in anderen Sprachen angelegte Vorstellung der Übersetzung als Transformation (etwa im Arabisch, Spanisch, Türkisch, Ungarisch, Chinesischen, Japanischen u.a.). Eine solche Bipolarität macht die verschiedenen Vokabulare vergleichbar und für die internationale Fachkommunikation übersetzbar. Genau diese Systematik provoziert den translationspolitischen Verdacht der fachsprachlichen und konzeptuellen Dissemination vom ,Zentrum“ in die ,Peripherie‘.

Der De-Westernizing-Debatte kommt zweifelsfrei das Verdienst zu, das methodologische Problem der Selektivität kulturspezifischer Begriffe bei der Bestimmung des Forschungsgegenstandes auf die Agenda gesetzt zu haben. Der von den De-Westernizern eingeschlagene Lösungsweg scheint nun der zu sein, eine neue Translationspolitik zu fördern, nach der translationsbezogene Reflexionsangebote der ,Peripherie‘ in das ,Zentrum' übersetzt werden sollen. ${ }^{13}$ Vor dem Hintergrund der Vergleichbarkeit der modernen Translationsvokabulare über die Sprach- und Kulturgrenzen hinweg wird häufig weit in historische, möglichst exotische Translationskulturen zurückgegangen, um die dort verwendeten translationsbezogenen Sprachbilder zu rekonstruieren. Als Indikator für die Tauglichkeit fremder translationskultureller Sprachspiele (Wittgenstein) für die Erweiterung und Justierung der Translationsbegrifflichkeit hat sich wohl schon früher ihre metaphorische Exotik erwiesen, wie der Übersetzungshistoriker LIEVEN D'HULST beobachtet, denn

[...] l'usage des métaphores, originales‘ est rarement ressenti comme inadéquat, à la différence de celles, victimes de l'usure, que le chercheur recontre dans les theories anciennes. (1992: 47)

Die Hoffnung ist offensichtlich, dass über die Rekonstruktion der vorwissenschaftlichen Beschreibungen von Translation die ursprüngliche Reflexionstradition einer Translationskultur zu Tage gebracht werden kann, die heute von einer meistens aus dem Englischen übersetzten Theoriesprache überlagert zu sein scheint. Daher wird bei solchen auf Internationalität abzielenden Studien meist nicht die aktuelle Begriffsdiskussion ,fremder Translationsdiskurse analysiert, sondern in der Vergangenheit liegende, mithin historische Denktraditionen. Auch auf der Ebene der Begriffs- und Theoriebildung werden um es salopp zu formulieren - ,Antiquitäten“ gesammelt.

13 Die in den letzten Jahren in den ,Flaggschiffen“ der translationswissenschaftlichen Verlagslandschaft (John Benjamins, St. Jerome, Routledge) erscheinenden Monographien, Editionen und Fachjournalen zeugen von dieser Programmatik. 
In der Überzeugung, dass Translation angesichts ihrer (ursprünglich) heterogenen Erscheinungsform zu komplex sei, um unter einen Begriff gefasst werden zu können, und im Bemühen der phänomenalen Heterogenität zu entsprechen, werden (metaphorische) ,Reflexionsbruchstücke' aus aller Welt zusammengetragen (ST. ANDRÉ 2010a, 2010b; TYMOCZKO 2007, 2009, 2014). Das methodologische Problem der unsachgemäßen Reduktion der Vielfältigkeit translatorischer Phänomene zu wenigen translationswissenschaftlichen Forschungsgegenständen durch eine bestimmte fachspezifische Begrifflichkeit soll offenbar dadurch aufgehoben werden, dass die Kulturspezifik der Reflexionsangebote aus der Peripherie bei der Übersetzung erkennbar bleibt. Die Begriffsarbeit, die die Translationswissenschaft leisten müsste, um ihren Gegenstandsbereich international $\mathrm{zu}$ integrieren, kann aber nicht durch das Zusammentragen historischer Sprachbilder und ihre Interpretation ersetzt werden. Es müsste zunächst rekonstruiert werden, welche begriffliche Ausarbeitung sich aus diesen Bildern heraus in den jeweiligen translationswissenschaftlichen Diskursen entwickelt haben. Die internationale Integration des Gegenstandsbereichs kann gerade nicht im Bezugsrahmen nationaler Translationskulturen verbleiben, möchte man nicht einer Provinzialisierung des Translationsdiskurses Vorschub leisten, die das Ziel der Internationalisierung des Fachs gerade verfehlen würde. Die Internationalisierung des Forschungsgegenstandes, würde vielmehr bedeuten, an der Extension und Intension des Translationsbegriffs zu arbeiten, um das Feld der Translationswissenschaft zu öffnen statt es zu fragmentieren. Um gleich einem Missverständnis zuvorzukommen: es geht mir nicht um die Einebnung aller kulturgebundenen Translationskonzepte zugunsten einer universalistischen Begriffsbestimmung. Eine auf Internationalität des Bezugsgegenstandes abzielende Begriffsarbeit müsste aber gerade im Lichte der Kenntnis möglichst vieler kulturspezifischer Translationskonzepte und -praktiken folgende Fragen zu ihrer dauerhaften Aufgabe machen: Welche normativen Erwartungen an Translation sind über die Grenzen unterschiedlicher Translationskulturen hinweg vergleichbar? Das heißt, welche Kriterien können (bis auf weiteres) für konstitutiv gelten für eine allgemeine Bestimmung von Translation und welche Bestimmungskriterien sind hingegen charakteristisch für spezifische Translationskulturen? Die Aufgabe einer internationalen Begriffs- und Theoriearbeit bestünde demnach darin, auf der Grundlage der Datensammlung gemeinsam den Haushalt der empirischen und der konstitutiven translationswissenschaftlichen Grundbegriffe neu zu ordnen, um eine effiziente translationsbegriffliche ,Falle“ im Sinne Blumenbergs zu erarbeiten. Effizient sind diese Instrumente nicht durch ihre Grenzenlosigkeit, sondern durch ihren passgenauen Zuschnitt und ihre gegenstandsbezogene, strukturierende Funktion.

\section{Zum Schluss: Internationalisierung der Forschungsgemeinschaft}

Wenn der De-Westernizing-Diskurs in seinem Bemühen, gegen die konzeptionellen Homogenisierungstendenzen anzugehen, möglichst weit in ,fremde ${ }^{6}$ translationskulturelle Geschichten zurückgeht, um originäre Übersetzungskonzepte zu rekonstruieren, dann wirkt dies angesichts der heutigen Ubiquität einer transfer- und originalfixierten 
Translationsvorstellung nicht nur wie ein Kampf gegen Windmühlen. Vielmehr erweisen sich diese Bemühungen als ein anachronistisches Unterfangen. Denn viele der mit großem Engagement analysierten historischen Translationskonzepte sind heute für die beobachteten Übersetzungskulturen selbst weder von translationspraktischer noch von transaltionswissenschaftlicher Relevanz (HELLER 2017a). Darüber hinaus droht der De-Westernizing-Diskurs im Interesse der Überwindung der, westlichen“ Originalfixierung und seines Essentialismus auf der Suche nach genuiner Alterität, seinem eigenen Essentialismus anheim zu fallen. Denn Überbetonung der Differenz und die voreilige Erklärung ihrer Überlagerung mit Verweis auf unidirektionale Transferprozesse vom ,Westen“ in den Rest der Welt setzt voraus, dass analytische Konzepte und Forschungsperspektiven unveränderliche Entitäten sind. Was wir aus einer sorgfältigen Rekonstruktion historischer Translationskulturen und deren sich wandelnden Begriffe aber gerade lernen, ist die Notwendigkeit der Beschreibung ihrer Historie als eine komplexe Übersetzungsgeschichte, die den Rekurs auf einen originären Begriff außerordentlich problematisch macht. Ich möchte dies zum Schluss an einem Beispiel aus der aktuellen chinesischen Terminologie veranschaulichen.

Der heute gebräuchliche chinesische Ausdruck für, Zielkultur'目的(语)文化 (mùdi(yŭ) wénhuà) erscheint, wenn als Einheit betrachtet, als eine typisch westliche Importware. Denn in 目的 (mudi - Ziel) manifestiert sich eindeutig die zielmetaphorische Terminologie europäischer Fachsprachen (Ausgangs-/Zieltext; source/target text; testo di partenza/di arrivo, texte de départ/d'arrivée, testo inicial/de destino). Der Terminus verweist auf die Übersetzungspolitik innerhalb des chinesischen Translationsdiskurses der 1980er und 90er Jahre, einer Blütezeit der Übersetzung. Den in dieser Zeit verfolgten allgemeinen Modernisierungsbestrebungen entsprechend war die Translationspolitik vornehmlich Ausgangssystem-orientiert. Entsprechend großen Einfluss hatte die Rezeption westlicher Theorien und -modelle auf die chinesische Übersetzungsdebatte. ${ }^{14}$ Tragen wir allerdings die historischen Schichten ab, indem wir die ,gewohnte' Wort-gebundene Übersetzungseinheit 目的(语)文化 in seine einzelnen Elemente zerlegen, wird sichtbar, dass es sich bei diesem Terminus nicht um eine kompakte unidirektional transferierte Einheit handelt, sondern dass diese über verschiedene Translationsprozesse aus unterschiedlichen Richtungen erst zu einer Einheit zusammengewachsen ist. Der Terminus ist nämlich aus Übersetzungen sowohl aus dem Westen als auch aus dem Osten zusammensetzt, denn 文化 (wenhua - Kultur) ist eine Übersetzung des japanischen Terminus für ,Kultur'. Bei näherer Betrachtung hat sich in diesem Terminus also neben der Westorientierung auch die translatorische Orientierung nach Japan vergegenständlicht. Der japanische Terminus selbst ist wiederum aus den Übersetzungsbemühungen des englischen sozial- und kulturwissenschaftlichen Begriffs ,culture' entstanden und zwar unter Zuhilfenahme des chinesischen Schriftzeichens 文 (wen - ursprünglich: ,gekennzeichnete Linie'; Schrift, Sprache). 文 hat seine Ursprünge in einem Piktogramm, das einen auf der Brust tätowierten Menschen darstellt. 化 (hua - verwandeln, zu etwas machen, zu etwas werden) verweist mit dem Radikal $\uparrow$ ebenfalls auf den Menschen. Über die Seg-

14 Für eine aufschlussreiche Rekonstruktion der Entwicklung des modernen Translationsdiskurses in China siehe CHAN (2004). 
mentierung der terminologischen Einheit in ihre kleinsten bedeutsamen Teile erkennen wir also auch, was mit dem translatologischen Massenimport aus dem Westen nicht in den chinesischen Diskurs übersetzt wurde, nämlich der essentialistische Kulturbegriff, so wie er in der westlichen Übersetzungswissenschaft lange tradiert worden ist. Genauer genommen ist mit 文化 nämlich ein Kulturbegriff bezeichnet, der sich unterscheidet von demjenigen, gegen dessen territoriale und nationale Implikationen die moderne ,westliche‘ übersetzungswissenschaftliche Theoriebildung glaubt ankämpfen zu müssen. Der chinesische Kulturbegriff verweist auf den Menschen als Kulturschaffenden, auf seine schöpferische Kraft und auf den transformativen Charakter von Kultur. Entsprechend dieses Bewusstseins über die Plastizität und Dynamik der Kultur stand auch die Frage nach der kultur- und sprachinnovativen, aber auch -dekonstruktiven Kraft der Übersetzung und ihre Instrumentalisierbarkeit schon sehr früh auch im Zentrum des modernen chinesischen Translationsdiskurses. Die translatorischen Einflüsse auf die Terminologiebildung in der übersetzungswissenschaftlichen ,Peripherie“ mögen also groß sein, doch verleitet die Vergleichbarkeit der Wortkörper dazu, über die Heterogenität begrifflicher Fundamente hinwegzusehen. Umgekehrt verführt die Verschiedenheit der Wortkörper und Metaphern dazu, vergleichbare (vergangene und gegenwärtige) Problemlagen zu übersehen (HELLER 2017a).

Es ging mir bei dem eben entwickelten Beispiel nicht um die Illustration einer fundierten und sorgfältigen Begriffsanalyse, die der Internationalisierung des Theoriediskurses zuträglich(er) wäre. Mit diesem Beispiel sollte vielmehr gezeigt werden, wie problematisch die essentialistische Unterscheidung ,originär" vs. ,importiert' ist, entlang derer sich die De-Westernizing-Debatte bei der internationalen Bestandsaufnahme analytischer Instrumente orientiert: Ist nun 目的文化 als ein westlicher/zentraler/importierte Begriff zu klassifizieren oder als ein nicht-westlicher/peripherer/originärer? Oder kann man den Begriff, um seiner Hybridität gerecht zu werden, in seine einzelnen Komponenten zerlegen, um diese ihren ,ursprünglichen' Reflexionskulturen zuzuordnen, um dann wiederum eine quantitative mithin objektive Einschätzung des ,Originalitätgrades' des Konzepts als Ganzes vorzunehmen? ${ }^{15}$ Wäre überhaupt die Feststellung, dass ein Begriff nicht importiert ist, ein Garant dafür, dass dieser Begriff in seiner ,natürlichen` Diskursumgebung besonders leistungsfähig ist bzw. leistungsfähiger als importierte Begriffe? Anders formuliert: was erwarten wir eigentlich von der Kategorisierung analytischer Entitäten in ,originär" und ,importiert' und welchen Beitrag soll eine solche Kategorisierung zur Internationalisierung der Begriffsbildung leisten? Meine Fragen zielen weder darauf ab, den Einfluss der Translationspolitik im Bereich der translationswissenschaftlichen Fachkommunikation herunterzuspielen. Noch möchte ich den Erkenntniswert differenzempfindlicher begriffshistorischer Untersuchungen in Abrede stellen. Wenn aber eine solche begriffshistorische ,Aufarbeitung' als ein Beitrag zur Internationalisierung der aktuellen Translationswissenschaft verstanden werden will, dann muss sie sich erstens

15 Gerade solche Fälle, bei denen die translatorischen Aneignungsprozesse nicht nur über die offensichtlichen sprachlichen, kulturellen und akademischen Grenzen, sondern auch über deren Dimension in Gestalt schriftlicher Notation (Alphabet vs. Sinographeme) hinweg geschehen, veranschaulichen das Aporetische solcher Zurechnungsversuche. 
von den derzeitigen Musealisierungstendenzen befreien. Eine zielführende begriffshistorische ,Aufarbeitung، müsste sich zweitens auch von ihrer normativen Kategorisierung befreien, nach der die wissenschaftspraktische Validität von Begriffen entlang der Unterscheidung ,originär' vs. (aus abendländischen Reflexionstradition), importiert' beurteilt wird. ${ }^{16}$ Denn diese Kategorisierung hat einen eigentümlichen paternalistischen Nebeneffekt: Sie ordnet die internationale Forschungsgemeinschaft gewissermaßen vor, indem sie bereits im Vorfeld die Positionen, d.h. die legitimen Positionen und Ansprüche der Diskussionspartner festlegt:

- Die „periphery researcher“, wie SUSAM-SARAJEVA (2002) sie nennt, werden darauf verpflichtet, ihre historischen indigenen Translationskonzepte zu profilieren, denn ,[t]hese researchers are ,educated away“ from their own culture and society“. (ibid.: 199, vgl. ä. CHEUNG 2005: 38 und TYMOCZKO 2009: 417f.) Sie sollten sich lieber auf ihre eigenen, selbst entwickelten Konzepte besinnen, statt das ,Zentrum' mit empirischem Material zu beliefern (SUSAM-SARAJEVA 2002: 204). Das Arbeiten mit Konzepten aus der abendländischen Tradition wird demgegenüber umstandslos als Irrweg oder als Akt der „Selbstkolonialisierung“ (LIU 1995: 236) und nach Anerkennung heischenden Nachahmung (SUSAM-SARAJEVA 2002: 198) diskreditiert.

- Die Forscherinnen aus dem translationswissenschaftlichen Zentrum werden hingegen darauf verpflichtet, ihre begrifflichen und theoretischen Gewohnheiten (vor allem hinsichtlich ihrer eurozentristischen Implikationen) zu hinterfragen und sich vom konzeptionellen Angebot aus der ,Peripherie“ inspirieren zu lassen (SUSAM-SARAJEVA 2002; TYMOCZKO 2006, 2009, 2014).

Unter dem Deckmantel der mitunter gönnerhaft anmutenden Forderung, „Toleranz“ aufzubringen für ,alternative“ Reflexionsstile (so z.B. SUSAM-SARAJEVA 2002: 204), werden bestimmte Translationskulturen zur kulturellen ,Selbstverteidigung“ genötigt und auf eine Reflexionstradition zurückgedrängt, die nicht ihrem aktuellen Forschungsstand entspricht. Die Historisierung von Translationskulturen verführt gewissermaßen dazu, bestimmte Forschungseinstellungen als ,natürlich“ oder ,natürlicher und darum als konservierungspflichtig darzustellen. Ein internationaler auf die Weiterentwicklung der aktuellen Begriffs- und Theoriebildung abzielender Diskurs muss dagegen auch für aktuelle Überzeugungen, Themen und Probleme geöffnet werden, auch wenn diese unter Umständen nicht den Differenzerwartungen mancher internationalizer entsprechen. Damit soll der Erkenntniswert historischer Arbeiten für die aktuelle Theoriediskussion nicht in Abrede gestellt werden. Eine zielführende historische Aufarbeitung bestimmter Translationskulturen müsste die ,exotisierten’ zeitgenössischen Forschungsgemeinschaften aber zu mindestens zwei Fragen zu Wort kommen lassen: erstens zu der Frage, welche Relevanz die interessierten vorwissenschaftlichen Reflexionstraditionen für sie selbst bei ihrer wissenschaftlichen Begriffsbildung haben; zweitens zu der Frage, wie die ,Aneignungsprozesse',fremdkultureller' Konzepte von den betreffenden Wissenschaftskulturen selbst gerechtfertigt, d.h. für sinnvoll erachtet oder problematisiert werden.

16 Für eine Kritik an der Bewertung theoretischer Angebote nach ihrer kulturellen Herkunft siehe auch CHESTERMAN (2014). 
Die primäre Bedingung für das Gelingen einer Internationalisierung der Fachdiskussion wäre damit, zunächst einmal einen ergebnisoffenen internationalen Dialog zu ermöglichen - damit ist sehr viel mehr gefordert als ,nur die sprachliche Ermöglichung des Gesprächs durch Translation. In einem solchen idealen Dialog sind nämlich weder die Teilnehmer vorbestimmt, noch die Gesprächsrollen verteilt, es sind keine Position festgelegt, noch sind spezifische Positionen a priori als mehr oder weniger ,valid', ,sachdienlich“ oder ,konstruktiv‘ kategorisiert. Idealerweise ergeben sich die Positionen gerade erst im Laufe einer Diskussion, in der argumentative Geltungsansprüche wechselseitig auf die Probe gestellt werden. Vor dem Hintergrund eines so verstandenen internationalen Dialogs wird ersichtlich, weshalb es der Translationswissenschaft nicht gelingen wird, den Vorwurf mangelnder Sensibilität für kulturelle und sprachliche Differenzen in der Fachdiskussion allein durch die Ausweitung des ,Jagdgebiets“ nach translatologischen Sprachbildern zu entschärfen. Zum einen, weil eine auf ,genuine‘ Alterität fixierte Suche den Blick auf den grundsätzlich translatorischen Charakter der Begriffsbildung verstellt (dies konnte weiter oben an der chinesischen Terminologie veranschaulicht werden). Zum anderen aber auch, weil der vom Exotikhunger Getriebene eigentlich nicht das Gespräch, sondern vor allem nach Differenz sucht.

\section{Literaturverzeichnis}

ALFER, Alexandra/Zwischenberger, Cornelia: Translaboration: Exploring Collaboration in Translation and Translation in Collaboration. In: Target (= Special Issue). Erscheint 2020.

BENNETT, Karen (2007): Epistemicide! The Tale of a Predatory Discourse. In: The Translator 13:2 (= Special Issue: Translation and Ideology: Encounters and Clashes), S. 151-169.

BENNETT, Karen (2015): Towards an epistemological monoculture: mechanisms of epistemicide in European Research Publication. In: Plo, Ramón/Pérez-Llantada, Carmen (Hrsg.): English as an Academic and Research Language. Berlin: de Gruyter Mouton (English in Europe 2), S. 9-35.

BISTUÉ, Belén (2017): On the Incorrect Way to Translate: The Absence of Collaborative Translation from Leonardi Bruni's De interpretatione recta. In: Cordingley, Anthony/Frigau Manning, Céline (Hrsg.): Collaborative Translation: From the Renaissance to the Digital Age. London: Bloomsbury, S. 33-48.

BOYDEN, Michael (2011): ,How eurocentric is Europe?‘ The challenge of linguistic justice theory to translation studies. In: Translation and Interpreting Studies 2 (=Sonderheft: Eurocentrism in Translation Studies), S. 174-188.

BLUMENBERG, Hans (2007): Theorie der Unbegrifflichkeit [aus dem Nachlaß hrsg. v. Anselm Haverkamp]. Frankfurt am Main: Suhrkamp.

CHAN, Tak-Hung Leo (2004): Twentieth-Century Chinese Translation Theory. Modes, issues and debates. Amsterdam/Philadelphia: John Benjamins (Benjamins Translation Library 51).

CHESTERMAN, Andrew (2014b): Translation Studies Forum: Universalism in translation studies. In: Translation Studies 7:1, S. 82-90.

CHEUNG, M. (2005): 'To translate' means 'to exchange? A new interpretation of the earliest Chinese attempts to define translation ('fanyi'). In: Target 17:1, S. 27-47. 
CHEUNG, Martha P. Y. (2006): An Anthology of Chinese Discourse on Translation. Volume 1: From Earliest Times to the Buddhist Project. Manchester/Kinderhook: St. Jerome.

CHEUNG, Martha P. Y./NEATHER, Robert (2017): An Anthology of Chinese Discourse on Translation Vol. 2. From the Late Twelfth Century. Abingdon/New York: Routledge.

CORDINGLEY, Anthony/FRIGAU Manning, Céline (2017): Collaborative Translation: From the Renaissance to the Digital Age. London: Bloomsbury.

D’HULST, Lieven (1992): Sur le rôle des métaphores en traductologie contemporaine. In: Target 1, S. 33-51.

DIZDAR, Dilek (2009): Translational transitions: ,Translation Proper' and Translation Studies in the Humanities. In: Translation Studies 2:1 (= Special issue: The Translational Turn. Hrsg. v. Doris Bachmann-Medick), S. 89-102.

FLECK, Ludwik (1979/1935): Genesis and Development of a Scientific Fact [hrsg. v. Thaddeus J. Trenn und Robert K. Merton; Vorwort v. Thomas S. Kuhn]. Chicago/London: The University of Chicago Press.

FOLENA, Gianfranco (1991): Volgarizzare e tradurre. Turin: Einaudi.

GIPPER, Andreas (2017): Von der Translatio zur Traductio. Zur problematischen Entdeckung des Kulturfaktors beim Übersetzen im italienischen Frühhumanismus. In: Heller, Lavinia (Hrsg.): Kultur und Übersetzung. Studien zu einem begrifflichen Verhältnis. Bielefeld: Transcript, S. 13-36.

GOEZ, Werner (1985): Translatio imperii. Ein Beitrag zur Geschichte des Geschichtsdenkens und der politischen Theorien im Mittelalter und in der frühen Neuzeit. Tübingen: Mohr.

HELLER, Lavinia (2017a): Translation zwischen alltäglicher Performanz und wissenschaftlichem Skeptizismus. In: Iwawaki-Riebe, Toyomi (Hrsg.): Sprache - Übersetzung - Welt(en). Möglichkeiten des Japanischen im globalen Zeitalter. Würzburg: Königshausen \& Neumann, S. 25-50.

HELLER, Lavinia (2017b): Eulen nach Athen? Provokation und Reflexionsanstöße des translational turn der Kulturwissenschaften für die Translationstheorie. In: Heller, Lavinia (Hrsg.): Kultur und Übersetzung. Studien zu einem begrifflichen Verhältnis. Bielefeld: Transcript (Inter-kulturalität. Studien zu Sprache, Literatur und Gesellschaft), S. 93-115.

HOLMES, James S. (1988): The future of translation theory: a handful of theses. In: Holmes, James S./van den Broeck, Raymond (Hrsg.): Translated! Papers on literary translation and Translation Studies, S. 99-102.

HOLZ-MÄNTTÄRI, Justa (1984): Translatorisches Handeln. Theorie und Methode. Helsinki: Suomalainen Tiedeakatemia.

INGGS, Judith/MEINTJES, Libby (2009): Translation Studies in Africa: central issues in interpreting and literary and media translation. London/New York: Continuum.

JANSEN, Hanne/WEGENER, Anna (2013): Authorial and Editorial Voices in Translation 1: Collaborative Relationships between Authors, Translators and Performers. Montréal: Éditions québécoises de l'œuvre.

KADE, Otto (1963): Aufgaben der Ubersetzungswissenschaft. Zur Frage der Gesetzmasigkeit im Ubersetzungsprozess. Leipzig: VEB Enzyklopadie.

KUHN, Thomas $\left({ }^{3} 1996 / 1968\right)$ : The Structure of Scientific Revolutions. Chicago: University of Chicago Press.

LIU, Lydia He (1995): Translingual Practice: Literature, National Culture, and Translated Modernity - China, 1900-1937. Stanford: Stanford University Press.

MARAIS, Kobus (2011): Can Tymoczko be translated into Africa? Refractions of research methodology in translation studies in African contexts. In: Southern African Linguistics and Applied Language Studies 29:3, S. 373-380. 
MARASSI, Massimo (2009): Leonardo Bruni e la teoria della traduzione. In: Studi umanistici piceni 29, S. 123-141.

O'HAGAN, Minako (2011): Translation as a social activity. Community translation 2.0. In: Linguistica Antiverpiensia New Series - Themes in Translation Studies 10. Brüssel: University Press Antwerp.

POPPER, Karl R. (1972): Objective knowledge: An evolutionary approach. Oxford: Oxford University, S. 341-362.

PRUNČ, Erich (2008): Zur Konstruktion von Translationskulturen. In: Larisa Schippel (Hrsg.): Translationskultur - ein innovatives und produktives Konzept. Berlin: Frank\&Timme, S. 19-41.

RISKU, Hanna (2014): Translation process research as interaction research: From mental to sociocognitive processes. In: MonTI (Minding Translation/Con la traducción en mente), S. 331-353.

SATO-ROSSBERG, Nana/WAKABAYASHI, Judy (2012): Translation and Translation Studies in the Japanese Context. London/New York: Bloomsbury (Bloomsbury Advances in Translation).

SCHIPPEL, Larisa/ZWISCHENBERGER, Cornelia (2016): Going East: Discovering New and Alternative Traditions in Translation Studies. Berlin: Frank \& Timme Verlag.

SUSAM-SARAJEVA, Şebnem (2002): A 'Multilingual' and 'International' Translation Studies? In: Hermans, Theo (Hrsg.): Crosscultural Transgressions. Research Models in Translation Studies II. Historical and Ideological Issues. Manchester: St. Jerome, S. 193-207.

Translation and Interpreting Studies 2 (2011) (=Sonderheft: Eurocentrism in Translation Studies)

Translation Studies 7:1 (2014).

Translation Studies 7:3 (2014).

TYMOCZKO, Maria (2006): Reconceptualizing Translation Theory. Integrating Non-Western Thought about Translation. In: Hermans, Theo (Hrsg.): Translating Others. Volume 1. Manchester/Kinderhook: St. Jerome, S. 13-32.

TYMOCZKO, Maria (2009): Why Translators Should Want to Internationalize Translation Studies. In: The Translator 2 (= Sonderheft: Chinese Discourses on Translation. Positions and Perspectives), S. 401-421.

TYMOCZKO, Maria (2014): Cultural Hegemony and the Erosion of Translation Communities. In: Bermann, Sandra/Porter, Catherine (Hrsg.): A Companion To Translation Studies. Chichester: Wiley Blackwell, S. 165-178.

VERMEER, Hans Josef (2000): Das Übersetzen in Renaissance und Humanismus (15. und 16. Jahrhundert), Bd. 2. Der deutschsprachige Raum. Literatur und Indices. Heidelberg: TEXTconTEXT (TEXTconTEXT Wissenschaft 7).

WAGNER, Birgit/LUTTER, Christina/LETHEN, Helmut (2012): Übersetzungen. Zeitschrift für Kulturwissenschaften 2. Bielefeld: Transcript.

WAKABAYASHI, Judy/KOTHARI, Rita (2009): Decentering Translation Studies. India and beyond. Amsterdam/Philadelphia: John Benjamins (Benjamins Translation Library 86).

Univ.-Prof. Dr. Dipl.-Übers. Lavinia Heller / lavinia.heller@uni-graz.at

Karl-Franzens-Universität Graz, Institut für Theoretische und Angewandte Translationswissenschaft, Merangasse 70/I, A-8010 Graz, AT 
